\title{
Ethische Theorien als gedankliche Tools - die Care Ethics
}

\section{Rouven Porz}

PD Dr. phil., dipl. biol., Leiter der Fachstelle für klinische Ethik, Insel Gruppe AG, Mitglied der Redaktion Ethik

Die sogenannte Prinzipienethik ist die bekannteste ethische Theorie der westlichen Medizinethik. Es lohnt sich auch, alternative ethische Theorien im Spitalalltag zu verwenden, so z.B. die Care Ethics. Diese Theorie fokussiert auf die Beziehungen und Abhängigkeiten der Akteure, stellt den jeweiligen situativen Kontext in den Vordergrund der Aufmerksamkeit und bedient sich z.B. des Perspektivenwechsels, um die moralische Verantwortung der beteiligten Akteure zu analysieren.

In meiner Arbeit bin ich oft mit folgendem Vorurteil konfrontiert: $\mathrm{Zu}$ viel Theorie bringt nichts im klinischen Alltag. Ich sehe das ganz anders. Erstens, es gibt kaum eine Theorie, die nicht ursprünglich auf praktischen Erfahrungen beruht (und umgekehrt!). Und zweitens, ich finde, dass insbesondere die Gesundheitsfachpersonen, die sich in klinischen Ethikstrukturen (Ethikkommissionen, Ethikforen usw.) engagieren, unbedingt über theoretisches ethisches Wissen verfügen müssen; dies aber nicht, um bei diesem Wissen konzeptuell stehenzubleiben, sondern um es in gedankliche Methoden umwandeln zu können. Diese Methoden mögen helfen, schwierige Fälle reichhaltiger aus ethischer Sicht zu analysieren.

\section{Theorie und Praxis}

Ich plädiere also dafür, verschiedene ethische Theorien als gedankliche Leitlinien anzusehen, als Werk-

\footnotetext{
Les théories éthiques comme outils de pensée l'éthique de la sollicitude

Les "principes de l'éthique biomédicale» (de T. Beauchamp et J. Childress) constituent la théorie majeure de l'éthique médicale occidentale. Cependant, d'autres théories éthiques peuvent aussi s'avérer intéressantes pour le quotidien clinique, dont Care Ethics. Inspirée du féminisme, cette théorie met l'accent sur les relations et les liens d'interdépendance entre les acteurs, replace l'attention à autrui dans le contexte particulier et recourt notamment au changement de perspective pour analyser la responsabilité morale des acteurs impliqués.
}

zeuge (Tools), oder - um noch eine andere Metapher zu nutzen - als unterschiedliche Lesebrillen, welche die zu untersuchende Situation je nach Brille in unterschiedlichem ethischem Licht erscheinen lassen können. Davon handelt der vorliegende Text, und ich illustriere meine Thesen an der sogenannten Theorie der Care Ethics. Und noch kurz zur Rekapitulation: Die prominenteste medizinethische Theorie der westlichen Ethikwelt haben wir im letzten, dritten Teil dieser Se-

Die Sichtweise der Care Ethics fragt nicht so sehr nach unseren juristischen Verpflichtungen, sondern vielmehr nach unseren moralischen Verantwortungen.

rie schon kennengelernt, die Prinzipienethik [1]. Die Gesundheitsfachpersonen sollen aus Sicht ihrer Berufsrolle folgende Prinzipien anwenden, um ethisch korrekt zu handeln, bzw. um ethische Analysen und Gewichtungen vorzunehmen: Respekt vor der Patientenautonomie zeigen, fürsorglich im Wohle des Patienten handeln, möglichst nicht schaden und gerecht denken. Jetzt fehlt uns vorliegend eigentlich nur noch ein klinischer Fall. Dazu konstruiere ich bewusst einen - wie ich persönlich finde - ganz und gar «unlösbaren» Fall, dessen scheinbare Lösungswege (künstliche Ernährung, Sterbenlassen, Zwangsmassnahmen) in mir allesamt ein mulmiges moralisches Gefühl auslösen. Zur Konstruktion des Falles greife ich zurück auf Daten, Ausführungen und die Vorarbeit der Forschungsgruppe Psychiatrie-Ethik am Institut für Biomedizinische Ethik der Universität Zürich um den Arzt und Psychologen Manuel Trachsel [2]. Es geht um eine junge Anorexie-Patientin. 


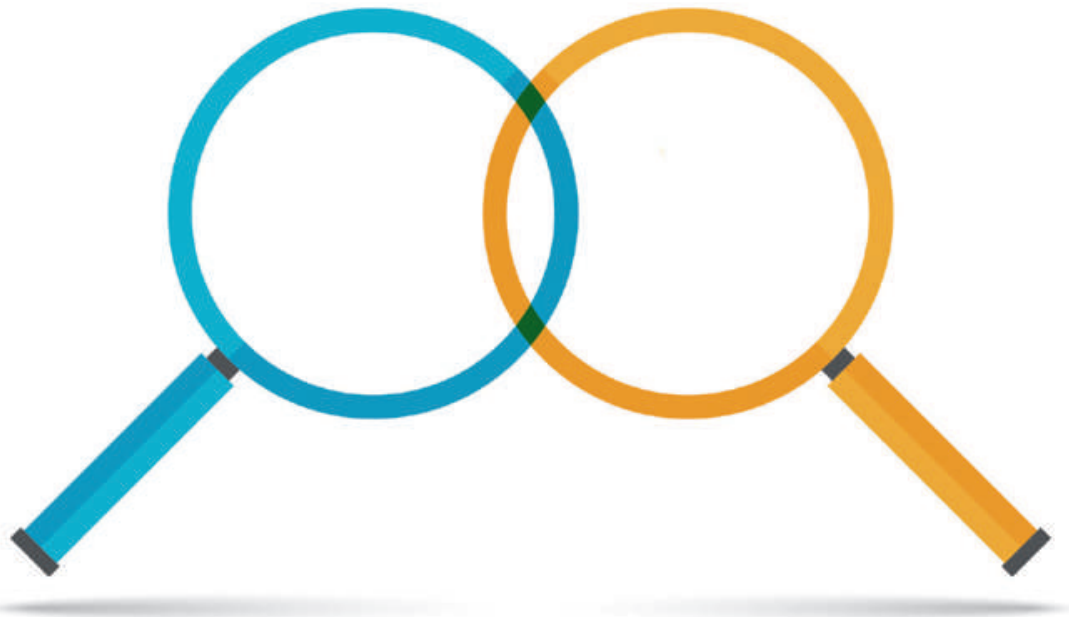

Problemsituationen sollten aus der Sicht verschiedener ethischer Theorien betrachtet werden - das hilft bei Analyse und Lösung.

\section{Fallbeispiel: 17-jährige Anorexie-Patientin}

Die 17-jährige Daniela S. leidet seit etwa fünf Jahren an Anorexia nervosa und wird aktuell in einer spezialisierten Universitätsklinik behandelt. Bei Aufnahme in die Klinik betrug ihr Gewicht 31,2 Kilogramm bei einer Körpergrösse von 1,60 Meter. Mit einem errechneten Body-Mass-Index von $12,2 \mathrm{~kg} / \mathrm{m}^{2}$ entspricht dies einem schweren Untergewicht. Den Aussagen der Mutter folgend habe Daniela Essen nie als etwas Genussvolles erlebt, sie sei auch in der Schule immer eine Aussenseiterin gewesen und mit 12 Jahren habe sie sich in einen zwei Jahren älteren Jungen verliebt, der ihre Gefühle aber gar nicht erwiderte. Er habe sogar gesagt, Daniela gefalle ihm nicht. Der Mutter zufolge sei dies der Auslöser der Essstörung gewesen. In den letzten fünf Jahren war sie schon zu insgesamt sechs Aufenthalten in einer spezialisierten Klinik hospitalisiert gewesen. Zweimal wurde sie schon gegen ihren Willen künstlich ernährt. Aktuell stellt das Untergewicht eine lebensbedrohliche Situation dar. Die Eltern drängen, doch baldmöglichst eine künstliche Ernährung einzuleiten. Daniela selbst sagt, sie sei nicht zu mager, und ihr grösster Wunsch sei es, endlich in Ruhe gelassen zu werden, keine Therapie mehr. Notfalls nehme sie den Tod in Kauf. Was soll das Behandlungsteam jetzt tun? Zur Klarstellung: In einer realen ethischen Fallbesprechung müssten nun zuerst einmal alle medizinischen, pflegerischen und vor allen Dingen psychologischen Darstellungen berücksichtigt und bewertet werden. Dann müsste aus juristischer Sicht die Urteilsfähigkeit von Daniela in Bezug auf die aktuelle Entscheidung gegen erneute künstliche Ernährung geprüft werden. Vielleicht ist sie in Bezug auf diese Entscheidung nicht urteilsfähig. Hier wäre auch zu bedenken, dass eben gerade das fortgeschrittene Stadium der Erkrankung zu weiteren kognitiven Einschränkungen führen kann. Eine Selbstgefährdung müsste dann bedacht werden, die eine stationäre Zwangseinweisung zur Folge haben könnte.

\section{Der ethische Konflikt aus Sicht der Prinzipienethik}

Für all diese Überlegungen haben wir hier vorliegend keinen Platz, und sie verschleiern auch nur den nicht aufzuhebenden prominenten ethischen Konflikt im Behandlungsteam: Der Respekt gegenüber Danielas Willensäusserungen steht im Widerspruch zu unseren Fürsorgebemühungen. Sie will nicht mehr ernährt werden. Wir wollen nicht, dass sie stirbt. Dies ist der Blick durch die Brille der Prinzipienethik.

Wie also weiterdenken? Man könnte die ethische Ebene ganz ausser Acht lassen und rein juristisch argumentieren: «Ja, wenn Daniela nicht mehr urteilsfähig ist, dann ist für uns bewiesen, dass sie auch nicht mehr autonomiefähig ist.» Ich finde, das ungute Bauchgefühl bleibt trotzdem zurück. Unser Problem ist ja nicht, Daniela in unterschiedliche Kategorien einordnen zu wollen, das könnte auch ein Roboter, unser Problem ist eher: Wie können wir Daniela möglichst viel Autonomiefähigkeit zugestehen, ohne dass wir als Gesundheitsfachteam auf unsere Fürsorgebemühungen verzichten müssen? Und schon stossen wir an die Grenzen der Prinzipienethik. Sie hat uns zwar konzeptuell gut geholfen, unser eher intuitiv wahrgenommenes schlechtes Bauchgefühl in eine gute ethische Frage zu übersetzen (Willensäusserung oder Fürsorge befolgen), aber sie hilft uns wenig bei der Suche nach möglichen Antworten auf diese Frage.

\section{Die Sicht der Care Ethics}

Ziehen wir eine neue Brille an, die Brille der Care Ethics. Ich strukturiere diese Einführung in die Care Ethics, indem ich nachfolgend fünf Charakteristika der Herangehensweise der Care Ethics unterscheide und aus meiner Sicht jeweils auf Danielas Fall projiziere [3]. Mir zumindest hilft die Care Ethics immer sehr gut beim Denken [4]. Meine Projektionen bleiben notwendigerweise subjektiv, aber die Lesenden sind gerne aufgefordert, ihre eigenen gedanklichen Welten zu erschliessen.

Serie zum Thema "Klinische Ethik neu gedacht» In dieser SÄZ-Serie geht es um die Anforderungen und Herausforderungen der klinischen Ethik als neuer Disziplin im Ge sundheitswesen. Die Beiträge sind bewusst subjektiv, interpretierend, aus Sicht eines klinischen Ethikers geschrieben und wollen zur Diskussion anregen. 


\section{Fünf Charakteristika der Care Ethics}

\section{Beziehungen}

Während die Prinzipienethik in ihrer Sichtweise davon ausgeht, dass Menschen grundsätzlich autonom handeln können und wollen, und dass wir alle unabhängig voneinander sind, betont die Sichtweise der Care Ethics eher das genaue Gegenteil: Wir sind immer in Beziehungen zueinander verhaftet, in (guten wie schlechten) Abhängigkeiten verstrickt. Zudem sind wir nicht alle gleich; ganz im Gegenteil, wir unterscheiden uns alle z.B. in Geschlecht, Hautfarbe, Herkunft, Möglichkeiten, Fähigkeiten und sozialer Stellung. Und wir unterscheiden uns in den Erfahrungen, die wir im Leben bislang gemacht haben. Um den gedanklichen Blick auf diese unterschiedlichen Beziehungen der Akteure zueinander zu legen, kann man als Frage formulieren: Wie lassen sich die Beziehungen/Abhängigkeiten/Erfahrungen der Akteure zueinander beschreiben?

Bezogen auf Daniela und ihr scheinbar kleines Beziehungsnetzwerk (nur Eltern, keine Freunde), würde ich in einer Fallbesprechung weiterfragen wollen, ob sie nicht noch andere Beziehungen auslebt, die für unser weiteres Vorgehen als Team einen Zugang zu ihr bieten könnten? Vielleicht virtuelle Beziehungen (Websites zu Anorexie, Blogging), vielleicht hat sie irgendein Vorbild? Irgendjemand, dem sie nacheifert? Die Abhängigkeit bezogen auf die Eltern (bzw. deren Abhängigkeit von ihr) tritt klar hervor, und ein weiterer wichtiger Punkt scheint mir ihre Erfahrung zu sein: Sie weiss, wovon sie redet, wenn sie sagt, sie wolle nie mehr wieder künstlich ernährt werden. Sie hat diese Erfahrung schon zweimal durchlebt. Vielleicht muss man ihre "gelebte Erfahrung» ernst nehmen, Urteilsfähigkeit hin oder her.

\section{Moralische Verantwortung}

Die Prinzipienethik geht in ihrer Sichtweise davon aus, dass Menschen Rechte haben. Im Gegensatz dazu betont die Sichtweise der Care Ethics nicht so sehr unsere juristischen Verpflichtungen, sondern vielmehr unsere moralischen Verantwortungen gegenüber unseren Mitmenschen, Freunden, Arbeitskollegen, Patienten usw. Natürlich lässt sich im Gesundheitswesen nie vermeiden, dass Verantwortung auch immer normative und durchaus auch rechtliche Konsequenzen nach sich zieht, aber es lohnt sich dennoch zu fragen: Wer trägt eigentlich die moralische Verantwortung in der Situation?

Diese Frage finde ich gerade in der Durchführung ethischer Fallbesprechungen sehr wichtig. Die Person, welche die moralische Verantwortung trägt, muss nicht dieselbe sein, die die juristische Verantwortung trägt. Falls Daniela tatsächlich urteilsunfähig ist, dann tragen ihre Eltern stellvertretend die juristische Verantwortung (mit den Ärzten, welche die Urteilsfähigkeit testieren). Ich würde aber argumentieren, und man mag mir widersprechen, dass es Daniela selbst ist, die die moralische Verantwortung trägt. Sie ist der ethischrelevante Akteur, die Ursache der Kausalkette, und dies muss klar herausgestellt werden (während man hier aus psychologischer Sicht gern anderer Meinung sein darf). Positiv daran ist, dass diese Sichtweise Daniela eine aktivere Rolle zuspielt. Man könnte sie gar danach fragen, was ihr die Verantwortung für ihre Eltern bedeutet.

\section{Kontext}

Die Prinzipienethik geht in ihrer Sichtweise davon aus, dass Situationen verallgemeinerbar und tendenziell vergleichbar sind. Im Gegensatz dazu betont die Sichtweise der Care Ethics die Besonderheiten im Kontext jeder neuen Problemsituation. Als Leitfrage ergibt sich: Was ist das Besondere am Kontext genau dieser Situation?

\section{Die Sichtweise der Care Ethics betont die Besonderheiten im Kontext jeder neuen Problemsituation.}

In komplizierten Fällen gibt es oft mehr Besonderheiten als Allgemeinplätze. Aber in Danielas Fall scheint es mir schon eine wichtige Komponente zu sein, dass die zunehmende Krankheit Auswirkungen auf ihre kognitiven Fähigkeiten hat / haben kann, und dass die Körperschema-Störung ein zentraler (für uns Aussenstehende kaum zugänglicher) Knackpunkt jeglichen logischen Denkens sein kann, will heissen: Selbst wenn Daniela «logisch» denkt, dann entspricht ihre Logik nicht notwendigerweise der unseren. Wir kommen auf keinen gemeinsamen Nenner, den man mit reiner Logik oder Rationalität erschliessen kann. Es müsste ein Weg gewählt werden, der reine Logik oder Rationalität umgehen kann.

\section{Giving voice}

In der Sichtweise der Care Ethics ist es wichtig, anzuerkennen, dass nicht jeder die gleiche Macht hat, die eigenen Interessen durchsetzen zu können. Manche Akteure unseres täglichen Alltags werden erst gar nicht ernst genommen oder es wird ihrer Meinung nicht ausreichend Bedeutung geschenkt. Die Care Ethics versteht sich deshalb auch als moralischer Anwalt der Vulnerablen, oft ausgedrückt in dem Slogan «giving voice». Auch für unsere Überlegungen zu Daniela 
könnte dieser gedankliche Anstoss wichtig sein, man kann fragen: Wer ist das schwächste Glied hier, welche Stimme wird am wenigsten gehört?

Das mag nun ein starke Überinterpretation von mir sein, subjektiv aus meinen Lebensumständen abgeleitet, und sie mögen selbst eine andere Spur verfolgen wollen, wenn vom vulnerabelsten Glied (ist es Daniela?) die Rede ist. Mir kommt aber nach der Fallbeschreibung unweigerlich die Frage in den Sinn: Was ist eigentlich mit dem Vater? Wieso taucht die Mutter so omnipräsent auf, aber dem Vater von Daniela wird kaum Platz gegeben. Müsste man diesem Gedanken noch nachgehen?

\section{Perspektivenwechsel-Geschichten ernst nehmen}

Die Care Ethics geht davon aus, dass die unterschiedlichen Akteure unterschiedliche Sichtweisen zu einer Situation haben können, unterschiedliche Geschichten: stories. Dieses Konzept des Perspektivenwechsels stellt eine direkte Schnittstelle zu weiterführenden ethischen Theorien dar, zur sogenannten narrativen und/oder hermeneutischen Ethik. Eine genauere Dar-

\section{Prinzipienethik und die Care Ethics sind wie zwei Seiten einer Münze.}

stellung dieser Theorien wäre sicherlich einen weiteren Text wert. In diesen Zugängen zur Ethik wird den Wert- und Normvorstellungen persönlicher Erzählungen besondere Beachtung geschenkt. Das heisst: Die Erzählungen werden so ernst genommen, wie sie sind, primär phänomenologisch, nicht psychologisch. Theoretische Grundlage ist die Idee, dass die von uns allen ständig durchgeführte Narration über/in unsere/-r persönliche/-n Lebenswelt geradezu konstitutiv für eben unsere Identitäten und unser Zusammenleben ist, und dass man aus diesen Narrationen die für den erzählenden Akteur relevanten Werte herauslesen kann [5]. Natürlich, die Geschichten, die jemand erzählt, müssen für Aussenstehende nachvollziehbar sein. Sie müssen sich an den alltäglichen Handlungen des Betreffenden messen lassen. Sie müssen diese Handlungen verständlich erklären und auch in ihren Gewichtungen bzw. Proportionen stimmig wirken [6]. Auf mich persönlich wirkt die Geschichte der Mutter nicht stimmig, wenn sie von dem jungen Mann erzählt, dem ihre Tochter nicht gefallen hat. Zu lange her, zu wenig Gewicht, ich bin skeptisch. Eine sehr starke Erklärungskraft hat allerdings für mich die finale Aussage von Daniela selbst: «[...] ihr grösster Wunsch sei es, endlich in Ruhe gelassen zu werden, keine Therapie mehr. Notfalls nehme sie den Tod in Kauf.» Der für sie wichtigste Wert ist klar herauszulesen: Sie will in Ruhe gelassen werden! Sie sagt nicht, dass sie sterben will. Aber wenn das Sterben eben die Norm ist, die aus ihrem wichtigsten Wert folgt, dann nimmt sie diese wohl oder übel in Kauf.

Trachsel schliesst [7], dass es eines kreativen (palliativen und/oder therapeutischen) Ansatzes bedarf, um die Autonomie der Patientin trotz fehlender Krankheitseinsicht zu gewährleisten.

\section{Sich ergänzende Theorien im Dienste der Problemlösung}

Ich wollte hiermit zeigen, dass eine alternative ethische Theorie wie die Care Ethics als zusätzliches gedankliches Hilfsmittel sehr gut in der Begründung eines solchen Mittelwegs helfen kann. Gleichzeitig wollte ich mit dieser Illustration zeigen, dass es in der Medizinethik nicht nur die Prinzipienethik gibt, ja, dass die Care Ethics sogar eine gute ergänzende und keine konkurrierende Theorie dazu ist. Für mich sind die Prinzipienethik und die Care Ethics wie zwei Seiten einer Münze. Man braucht sie beide, um in einer soliden ethischen Währung bezahlen zu können.

Danksagung

Ich danke Dr. Dr. Manuel Trachsel und Dr. Rebecca Ott für die hilfreichen Kommentare in der Durchsicht meines Textes.

Bildnachweis

Herminutomo | Dreamstime.com

\section{Literatur}

1 Porz R. (2015): Werte, Moralvorstellungen und Berufsrollen. In: Schweiz Ärztezeitung. 2015;96(42):1533-6.

2 Trachsel $\mathrm{M}$ et al. Compulsory treatment in chronic anorexia nervosa by all means? Searching for a middle ground between a curative and a palliative approach. In: The American Journal of Bioethics. 2015;15(7):55-6. Und: Trachsel M et al. (in print): Zwangsernährung oder Palliative Care bei chronischer Anorexia Nervosa? In: Torsten Moos et al. Randzonen des Willens, Entscheidungen und Einwilligungen in Grenzsituationen der Medizin.

3 Die Darstellung der Charakteristika ist inspiriert durch: Feder Kittay E. The concept of Care Ethics in Biomedicine. In: RehmannSutter et al. (eds). Bioethics in Cultural Context. Springer; 2006; S. 319-41.

4 Vgl. hierzu ähnlich: Wick A, Porz R. Implizite Machtstrukturen in der Psychiatrie - Eine Care Ethics Perspektive. In: Porz R et al. (Hg.) Die Macht der Fürsorge? TVZ; 2015. S. 195-209.

5 Vgl. Walker, MU. Moral understandings. A feminist study in Ethics. Oxford, University Press; 2007. Und: Porz R, Landeweer E, Widdershoven G. Theory and practice of clinical ethics support services: narrative and hermeneutical perspectives. Bioethics. 2011;25(7):354-60.

6 Lindemann H. Wrinkles in time: narrative approaches to ethics. von Pfleiderer G et al. (HG.) Zeithorizonte des Ethischen - Zur Bedeutung der Temporalität in der Fundamental- und Bioethik. Stuttgart: Kohlhammer; 2006. S. 123-32.

7 Vgl. Fussnote 2. 